\title{
A systematic review assessing non-pharmacological conservative treatment studies for people with non-inflammatory multi-joint pain: clinical outcomes and research design considerations
}

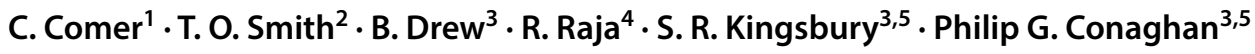

Received: 19 July 2017 / Accepted: 4 November 2017 / Published online: 16 November 2017

(c) The Author(s) 2017. This article is an open access publication

\begin{abstract}
To systematically review the evidence to determine the clinical outcomes and the important methodological quality features of interventional studies on adults with non-inflammatory multi-joint pain (MJP). Systematic search of published and unpublished literature using the databases: AMED, CINAHL, MEDLINE, EMBASE, psycINFO, SPORTDiscus, PEDro, OpenGrey, the EU Clinical Trials Register, World Health Organization International Clinical Trial Registry Platform, ClinicalTrials. gov and the ISRCTN registry (search: inception to 19th October 2017). All papers reporting the clinical outcomes of nonpharmacological interventions for people with non-inflammatory MJP were included. Studies were critically appraised using the Downs and Black Critical Appraisal and the TIDieR reporting checklists. Data were analysed using a Best Evidence Synthesis approach. From 3824 citations, four papers satisfied the eligibility criteria. Three studies reported outcomes from multidisciplinary rehabilitation programmes and one study reported the findings of a spa therapy intervention. All interventions significantly improved pain, function and quality of life in the short-term. There was limited reporting of measures for absenteeism, presenteeism and psychosocial outcomes. The evidence was 'weak', and due to a lack of controlled trials, there is limited evidence to ascertain treatment effectiveness. Design consideration for future trials surround improved reporting of participant characteristics, interventions and the standardisation of core outcome measures. There is insufficient high-quality trial data to determine the effectiveness of treatments for non-inflammatory MJP. Given the significant health burden which this condition presents on both individuals and wider society, developing and testing interventions and accurately reporting these, should be a research priority.

Registration PROSPERO (CRD42013005888).
\end{abstract}

Keywords Pain · Arthritis · Therapeutics $\cdot$ Clinical Trial $\cdot$ Research design

\section{Introduction}

C. Comer and T. O. Smith contributed equally to this work.

Philip G. Conaghan

p.conaghan@leeds.ac.uk

1 Extended Scope Physiotherapy Practitioner, Leeds Community Healthcare Musculoskeletal and Rehabilitation Services, Leeds, UK

2 Physiotherapy, School of Health Sciences, University of East Anglia, Norwich, UK

3 Leeds Institute of Rheumatic and Musculoskeletal Medicine, University of Leeds, Leeds, UK

4 Christchurch Hospital, Christchurch, New Zealand

5 NIHR Leeds Biomedical Research Centre, University of Leeds, Leeds, UK
Musculoskeletal pain presents a significant clinical challenge and is associated with a substantial health and social burden $[1,2]$. The majority of patients with musculoskeletal complaints experience pain at more than one joint $[3,4]$. Non-inflammatory multi-joint pain (MJP) represents a complex mix of osteoarthritis, back pain and soft tissue disorders [5]. It is associated with increased disability, depression and lost work productivity [6]. People with MJP may have a median of six painful joints [5] and evidence indicates that an increasing number of painful joints is associated with poorer physical and mental statuses [7, 8], increasing the risk of restrictions on both activity and social participation. The costs associated with MJP are significantly greater than 
those associated with low back pain alone, which costs the NHS approximately $£ 1700$ million annually [2].

Both clinical care and research have traditionally focused on treating single joint pain and disability [9], failing to recognize the impact of MJP on treatment choices and outcomes [10]. Whilst a recent survey suggests that the majority of general practitioners now treat MJP concurrently rather than focusing on a single joint [11], there remains uncertainty on how this should be operationalized, and what interventions should be used. To this end, the National Institute for Health and Care Excellence (NICE) recommended in their osteoarthritis guidelines [12] that trials to investigate interventions for the management of MJP should be a research priority.

Currently, only one systematic review has examined interventional trials for people with MJP [9], concluding that there is limited evidence to guide treatment choice. However, whilst all studies in this review investigated the effectiveness of an intervention package designed for people with MJP, in practice, participants were recruited into these trials with single joint pain in the hip, knee or hand joints [13-15] rather than targeting people with co-existing pain in two or more joints. Furthermore, this review paper was based only on studies which were multidisciplinary in delivery (two or more different health professional groups), were delivered in primary or community care settings, and were required to incorporate NICE recommended core treatments [12]. Consequently, it remains unclear whether outcomes for these interventions would differ with a true MJP population, and if there are other interventions which may be supported for this population when delivered by specific professional groups in secondary and primary care.

The purpose of this review is, therefore, to: (1) address this uncertainty and examine the current literature to determine the effectiveness of different interventions for people with non-inflammatory MJP, and (2) identify key research design features which should be considered when designing future trials on people with non-inflammatory MJP.

\section{Methods}

\section{Search strategy}

The primary search was of published literature searching the databases: AMED, CINAHL, MEDLINE, EMBASE, psycINFO, SPORTDiscus and PEDro. Secondary search strategies included searching the unpublished and grey literature databases: OpenGrey, the EU Clinical Trials Register, World Health Organisation International Clinical Trial Registry Platform (ICTRP), ClinicalTrials.gov, and the ISRCTN registry. All databases were searched from database inception to 19th October 2017, and performed by one reviewer (TS). The search strategy for the MEDLINE search (via Ovid) is presented in Table 1. This was modified for each individual database. The reference lists from all potentially eligible papers and review papers were reviewed. All corresponding authors from each included study were contacted to review the search results to identify any additional studies which may have been initially omitted.

\section{Eligibility criteria}

Studies were eligible if they satisfied the following criteria.

\section{Design}

Randomized or non-randomized trials presenting clinical outcomes for one or more defined interventions for the population of interest. Data on the location of intervention, who delivered it and the frequency to which it was provided was the minimum information required to be defined as an intervention. We excluded all basic science research and animal studies.

\section{Population}

Adults (16 years and over) with concurrent pain located at two or more joints e.g. ankle and hip. In accordance with Raja et al. [5] definition of MJP, we defined a joint-site as a region e.g. hand, foot, rather than by individual 'small'

Table 1 MEDLINE search strategy

1. joint diseases/
2. arthropathy.ti,ab
3. arthritis/
4. esp osteoarthritis/
5. (pain\$ adj3 (dual\$ widespread or many)).ti,ab
6. (pain\$ adj3 (number or one or two or three or four) adj3 (site\$ or
location $\$$ or area $\$$ or joint $\$$ )).ti,ab
7. (pain\$ adj3 (multi or multi?joint or multi?site or multi?focal)).
ti,ab
8. ((multi\$ or widespread or dual\$) adj5 (musculo?skelet $\$$ or joint\$)
adj5 (pain\$ or problem\$)).ti,ab
9. generalized osteoarthritis.ti,ab
10. generalized pain.ti,ab
11. widespread pain.ti,ab
12. musculoskeletal pain.ti,ab
13. widespread musculoskeletal pain.ti,ab
14. multisite musculoskeletal pain.ti,ab
15. multiple pain sites.ti,ab
16. regional pain.ti,ab
17. fibromyalgia/
18. fibromyalgia.ti,ab
19. OR/2-18
20. AND/1,19

2. arthropathy.ti,ab

3. arthritis/

4. esp osteoarthritis/

5. (pain\$ adj3 (dual\$ widespread or many)).ti,ab

6. (pain $\$$ adj3 (number or one or two or three or four) adj3 (site\$ or $\mathrm{ti}, \mathrm{ab}$ adj5 (pain\$ or problem\$)).ti,ab

10. generalized pain.ti,ab

11. widespread pain.ti,ab

12. musculoskeletal pain.ti,ab

13. widespread musculoskeletal pain.ti,ab

14. multisite musculoskeletal pain.ti,ab

15. multiple pain sites.ti,ab

16. regional pain.ti,ab

17. fibromyalgia/

20. AND/1,19 
joints such as 1st carpometacarpal and 5th proximal interphalangeal joint. It could include combinations of joint disorders including osteoarthritis, back pain and tendinopathy. Pain could be a self-reported and/or physical examinationbased diagnosis, but we excluded studies where MJP was diagnosed solely by radiological investigation. We excluded studies where participants reported pain in two or more locations without specific joint involvement such as fibromyalgia, myofascial pain, or widespread pain originating from soft-tissue/connective tissue disorders. "Methods" or "Results" section required to clearly indicate concurrent involvement of two or more joints. If the term "and/ or" for joint involvement was used in the methodology, the "Results" section must have described concurrent involvement of two or more joints. e.g. 'ankle and hip joint pain' or 'five percent of participants had pain in two joints'. However, we included studies which recruited people with MJP and non-MJP where the population with MJP was specifically identified and results for the cohort were reported separately, or where $90 \%$ or over of that cohort were diagnosed with MJP. We excluded all studies where participants had inflammatory arthropathies such as rheumatoid arthritis.

\section{Intervention}

Any non-pharmacological interventions or care pathways for the population of interest were included. This, therefore, included exercise and physical activity interventions, pacing and behavior modification interventions, psychological interventions, self-management programmes and device/assisted technologies. Packages of care which included one or more of these interventions were included. Interventions which comprised both a pharmacological and non-pharmacological treatment were included when the principle intervention was non-pharmacological and the pharmacological treatment an adjunct. We did not place a restriction on the frequency or intensity of an intervention, the location of delivery or who (which professionals) delivered the intervention.

\section{Comparison}

Any intervention or non-treatment control group was eligible as a comparator. Papers which did not include a comparator group (i.e. pre-post test design) were included.

\section{Outcome}

The a priori primary outcome measure was pain at 6-months post-commencement of the intervention. This could have been measured as part of a tool such as the Western Ontario and McMaster University Arthritis Index score (WOMAC) [16] or as a numerical rating scale (NRS) or visual analogue scale (VAS) pain score.
Secondary outcome measures were:

- Pain measured at other time-points,

- Physical function measured with tools such as the WOMAC [16], Knee Injury and Osteoarthritis Outcome Score (KOOS) [17] or Oswestry Disability Index scores (ODI) [18],

- Health-related quality of life measured with instruments such as the Short Form-12 (SF-12) [19], SF-36 [20], or the EQ-5D-5L [21],

- Anxiety and depression measured with tools such as the Hospital Anxiety and Depression scale (HADS) [22],

- Fear avoidance and kinesphophia measured with tools such as the Fear Avoidance Beliefs Questionnaire [23] and Tampa Scale of Kinesphobia [24],

- Self-efficacy measured with tools such as the General Self-Efficacy Scale [25],

- Work absenteeism and presenteeism.

Assessment intervals were defined as immediate-term (0-6 weeks), short-term (more than 6 weeks -3 months), mid-term (more than 3-12 months) and longer-term (more than 12 months).

\section{Publication}

We included all papers which reported eligible trials, irrespective of date of publication, language of publication, or where (geographically) the study was conducted.

\section{Study identification}

Three reviewers (RR, BD, CC) independently reviewed the titles and abstracts of all search results using the defined eligibility criteria. Full-texts for all papers deemed potentially eligible were gathered, and re-reviewed by the three reviewers (RR, BD, CC) for full eligibility. All papers satisfying the criteria and agreed between two or more reviewers were included.

\section{Data extraction}

Data extracted included: population characteristics definition of MJP; participant: age, gender, joint pain location, number of joint pain sites, BMI, SES group; country of trial origin; sample size; location of intervention delivery (i.e. primary or secondary care); intervention constituents i.e. treatment types, dose and frequency, self-management/ home management programme, co-interventions; control intervention constituents; outcome measures and followup intervals assessed; length of follow-up; clinical findings including effect size and intervention fidelity. All data were collected independently onto a pre-defined data extraction 
table by three reviewers (BD, CC, TS). Any disagreements were resolved through discussion.

\section{Critical appraisal}

To assess the quality of the current evidence and key design features for trials of people with MJP, all included studies were critically appraised using the Downs and Black Checklist [26]. This is a reliable and valid critical appraisal tool for non-randomised and randomised controlled trials [26]. It includes 27-items assessing: reporting, external validity, internal validity and power. To assess the reporting of study interventions we used the 12-item TIDieR checklist [27]. This assesses: intervention reporting by asking questions on: why, what (materials), what (procedure), who provided, how, where, when and how much, tailoring, modifications, how well (planned), how well (actual) [27]. The assessment for both checklists was independently performed by two reviewers (BD, CC).

\section{Data synthesis}

Study heterogeneity was assessed by examining the data extraction table. Due to between-trial variability in cohort participant's characteristics, interventions (experimental and control) and study design/processes, a meta-analysis was inappropriate [28]. Consequently, a narrative analysis of the data was performed using a Best Evidence Synthesis approach [29] where studies were graded as 'strong', 'moderate' or 'weak' as a judgement made by the three reviewers (CC, BD, TS) based on the Downs and Black assessment and the TIDieR evaluation.

\section{Results}

\section{Search strategy}

A summary of the search results is presented as Fig. 1. A total of 3824 citations were identified of which 126 were deemed potentially eligible and reviewed at full-text level. The reasons for exclusion included papers not reporting interventional studies $(n=102)$ or they did not include patients who met the a priori definition of MJP $(n=16)$, or were study protocols $(n=4)$. Of the full-text papers reviewed, four satisfied the eligibility criteria and were included.

\section{Characteristics of included studies}

The characteristics of the included studies are presented in Table 2. Three papers reported the findings from observational pre-test/post-test investigations $[6,10,30]$, whilst one paper reported a randomised controlled trial (RCT) [31]. Three studies presented data on multidisciplinary team (MDT) programmes for people with MJP [6, 10, 31]. Erol et al. [30] reported the clinical findings of a spa therapy intervention.

Two studies characterised and termed their cohorts as people with 'generalised osteoarthritis' [30, 31]. Lillefjell et al. [6] and Moradi et al. [10] termed this group 'chronic musculoskeletal pain' but provided specific definitions of joint involvement, thereby meeting the eligibility of MJP for this review. In all four studies, the definition of joint pain in two or more joints were specified (Table 2). Moradi et al. [10] and Lillefjell et al. [6] recruited people with both single and MJP. However, Moradi et al. [10] reported the findings of single- and MJP separately, whilst $94 \%$ of Lillefjell et al's [6] cohort were MJP and, therefore, met the eligibility criteria.

\section{Quality assessment}

A summary of the critical appraisal results is presented in Table 3. The randomised controlled trials presented with a moderate risk of bias, whilst the non-randomised controlled trials presented with 'low' quality evidence. In both, the TIDieR checklist assessment (Table 4) highlighted recurrent limitations in intervention reporting. The included studies were consistently poor in reporting materials and participant's role within and towards the intervention and its delivery (Item 3;25\%), the description of the intervention's activities (Item $4 ; 25 \%$ ), the dosage (Item 8; 0\%), the adaption or modification of the intervention (Item 9 and 10;0\%) and only Cuperuset al [31] reported the fidelity (Item 12; $25 \%$ ). Based on the quality assessment, seven key research design considerations where identified for consideration when designing future trials for people with MJP. These are presented in Table 5.

\section{Intervention 1: Multi-disciplinary programme}

Three studies presented data on MDT programmes in people with MJP $[6,10,31]$.

\section{Pain}

Two studies of moderate [31] and weak [6] evidence were available to support a significant decrease in pain at short-term follow-up. Cuperus et al. [31] reported a significant decrease in pain by 3.2 points at 6 -week followup. Lillefjell et al's [6] MDT programme reported a mean decrease in pain by 3.8 points $(p<0.05)$ at 5 -week followup. Moderate evidence was available from Cuperus et al. 


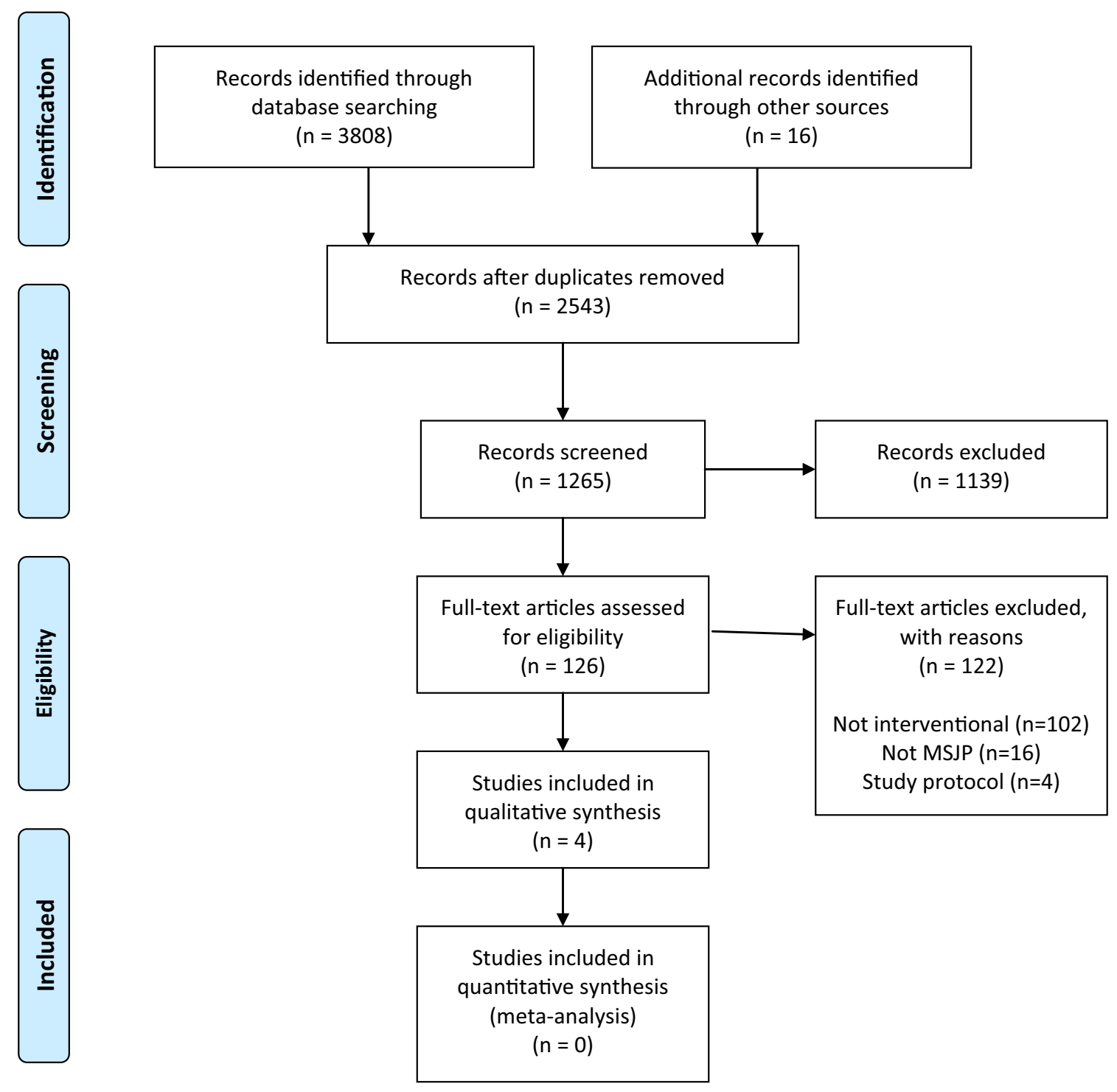

Fig. 1 PRSIMA flow-chart of search results

[31] of no statistically significant change in pain score for their telephone-based intervention (6 week difference: 0.96; 1-year: 0.76).

There was weak evidence from one study [10] to support a significant decrease in pain at mid-term follow-up (6 months). Moradi et al. [10] reported a mean decrease of 1.4 points $(p<0.001)$ for their dual-joint pain group, and 1.2 points for their MJP group $(p<0.001)$.

Two studies of moderate [31] and weak [6] evidence were available to support a significant decrease in pain at long-term follow-up. Cuperus et al. [31] reported a 2.8 point decrease at 1 -year $(p<0.05)$ for their face-toface MDT intervention and Lillefjell et al. [6] 5.7 points $(p<0.01)$ at long-term (57 week) follow-up.

\section{Physical function}

Two studies of moderate [31] and weak [6] quality were available to evaluate physical function. Cuperus et al. [31] reported a statistically significant increase in physical function for their cohort who received the face-to-face and telephone-based MDT interventions at 6 weeks (mean difference form baseline: 2.25 and 1.58 , respectively; $p<0.05)$. Similarly, Lillefjell et al. [6] reported a mean difference in COOP/ WONCA daily activity assessment but only by 0.10 points and 0.23 points at 5 and 57 weeks follow-up $(p<0.001)$.

There was weak evidence from one study [10] to support improvements at mid-term follow-up. Moradi et al. [10] reported a mean increase in SF-36 physical function of 17.1 


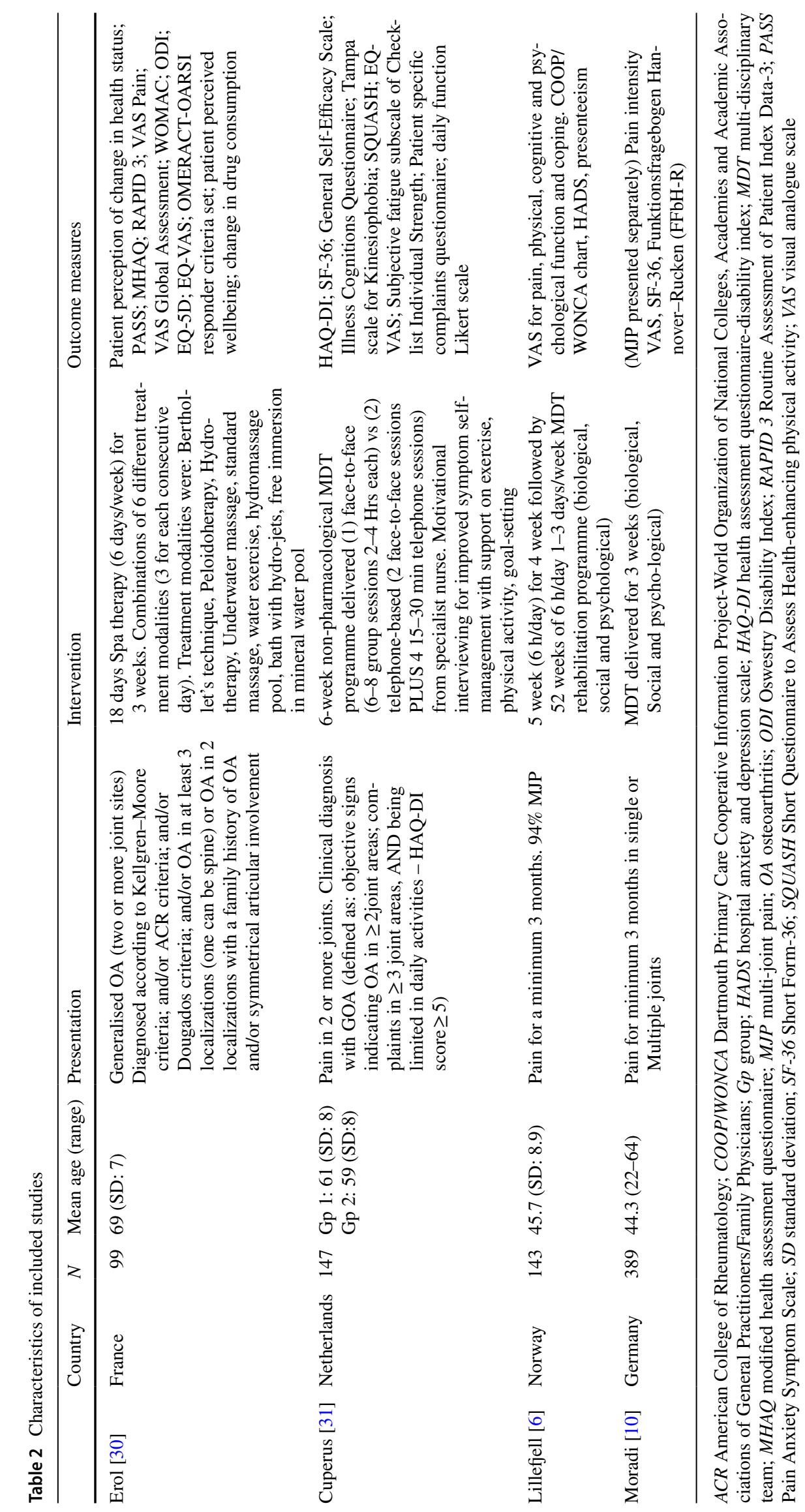




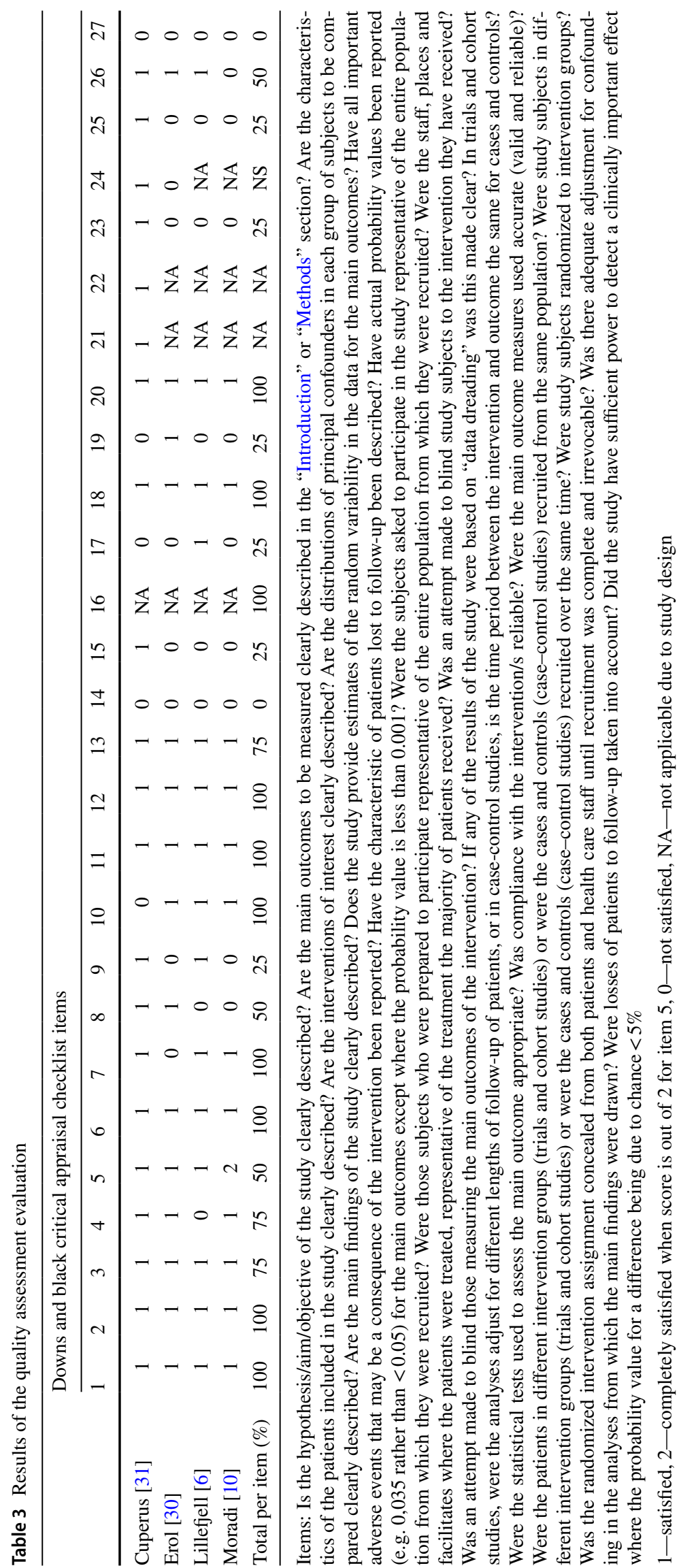


Table 4 TIDieR checklist for interventional reporting

\begin{tabular}{|c|c|c|c|c|c|c|c|c|c|c|c|c|c|}
\hline & \multicolumn{12}{|c|}{ TIDieR checklist items } & \multirow{2}{*}{$\begin{array}{l}\text { Total per } \\
\text { study } \\
(\%)\end{array}$} \\
\hline & 1 & 2 & 3 & 4 & 5 & 6 & 7 & 8 & 9 & 10 & 11 & 12 & \\
\hline Cuperus [31] & 1 & 1 & 0 & 0 & 1 & 1 & 0 & 0 & 0 & 0 & 1 & 1 & 50 \\
\hline Erol [30] & 1 & 1 & 1 & 1 & 1 & 0 & 1 & 0 & 0 & 0 & 1 & 0 & 58.3 \\
\hline Lillefjell [6] & 1 & 1 & 0 & 0 & 0 & 0 & 0 & 0 & 0 & 0 & 0 & 0 & 16.7 \\
\hline Moradi [10] & 1 & 1 & 0 & 0 & 0 & 1 & 0 & 0 & 0 & 0 & 0 & 0 & 25 \\
\hline Total per item (\%) & 100 & 100 & 25 & 25 & 50 & 50 & 25 & 0 & 0 & 0 & 50 & 25 & \\
\hline
\end{tabular}

Checklist items: Provide the name or a phrase that describes the intervention. Describe any rationale, theory, or goal of the elements essential to the intervention. Describe any physical or informational materials used in the intervention, including those provided to participants or used in intervention delivery or in training of intervention providers. Provide information on where the materials can be accessed (e.g. online appendix, URL). Describe each of the procedures, activities, and/or processes used in the intervention, including any enabling or support activities. For each category of intervention provider (e.g. psychologist, nursing assistant), describe their expertise, background and any specific training given. Describe the modes of delivery (e.g. face-to-face or by some other mechanism, such as internet or telephone) of the intervention and whether it was provided individually or in a group. Describe the type(s) of location(s) where the intervention occurred, including any necessary infrastructure or relevant features. Describe the number of times the intervention was delivered and over what period of time including the number of sessions, their schedule, and their duration, intensity or dose. If the intervention was planned to be personalised, titrated or adapted, then describe what, why, when, and how. If the intervention was modified during the course of the study, describe the changes (what, why, when, and how). Planned: If intervention adherence or fidelity was assessed, describe how and by whom, and if any strategies were used to maintain or improve fidelity, describe them. Actual: If intervention adherence or fidelity was assessed, describe the extent to which the intervention was delivered as planned points specifically in their dual-joint pain cohort and 9.2 points in their MJP cohort at 6-month follow-up $(p<0.001)$.

There was conflicting evidence from one study of moderate [31] and another of weak quality [6] regarding functional outcomes in the longer-term. Cuperus et al. [31] reported neither intervention provided statistically significant findings from baseline for this measure at 12 months (SF-36 physical function: mean difference from baseline: 1.58 points and 1.13 points; $p>0.05$ ). Lillefjell et al. [6] reported statistically significant improvements in physical function at longer-term follow-up assessments.

\section{Health-related quality of life}

One study [31] of moderate evidence supported improvements in quality of life using the EQ-VAS. Cuperus et al. [31] reported a significant improvement in quality of life in both intervention groups at 6 -week follow-up $(p<0.05)$, the magnitude of improvement being greater for the face-toface group (mean difference: 8.43 points) compared to the telephone-based intervention group (mean difference 5.22 points). At 12 months, this difference remained in the faceto-face group (mean difference $6.59 ; p<0.05$ ), but not in the telephone-based intervention group (mean difference 3.73 ).

\section{Anxiety and depression}

One study [6] of weak evidence assessed anxiety and depression at short and long-term follow-up using the HADS.
Lillejjfell et al. [6] reported that both anxiety $(p<0.05)$ and depression $(p<0.01)$ both significantly decreased at the 5 and 57-week follow-up intervals in this MDT intervention. This was not a large change. There was a mean reduction in anxiety by 0.18 points at 5 weeks, and 0.9 at 57 weeks. Similarly, there was a mean reduction in depression by 0.44 points at 5 weeks, and 0.95 at 57 weeks.

\section{Fear avoidance and kinesophophia}

There was moderate evidence from one study [31] showing there was no statistically significant difference in kinesophobia in either the face-to-face MDT intervention or telephonebased intervention at 6 weeks or 12 months $(p>0.05)$.

\section{Self-efficacy}

There was moderate evidence from one study [31] reporting no statistically significant difference in self-efficacy when evaluated using the General Self-Efficacy Questionnaire in either the face-to-face MDT intervention or telephone-based intervention at 6 weeks or 12 months $(p>0.05)$.

\section{Missing outcomes}

No studies reported findings of fear avoidance, absenteeism or presenteeism for either of the three MDT intervention papers. 


\section{Intervention 2: Spa therapy}

One study presented outcomes of a spa therapy intervention for people with MJP [30]. Using a Best Evidence Synthesis approach, the outcomes from this study were classified as 'weak' evidence.

\section{Pain}

Whilst there was a statistically significant improvement in pain from baseline to post-treatment (4.8 to $3.7 ; p<0.01$ ), this was not statistically significant at mid-term ( 8 months) follow-up (4.8 to $4.5 ; p=0.15$ ).

\section{Physical function}

There was a statistically significant improvement in physical function when measured using the WOMAC subsection from baseline to post-treatment $(19.5-15.2 ; p<0.01)$. This was not statistically significant at mid-term ( 8 months) follow-up (19.5-19.7; $p=0.76)$. The Oswestry Disability Index decreased in the immediate-term ( 3 weeks) from 25.4 to $20.8(p<0.01)$ and at the mid-term but only differed by two points $(p=0.04)$.

\section{Health-related quality of life}

Immediate-term (3-week commencement of treatment), 33\% reported an acceptable symptom state (achieved PASS). Mid-term (8-month post-commencement of treatment) this increased to $75 \%$. There was a significant increase in the Modified Health Assessment Questionnaire $(p=0.03)$, Routine Assessment of Patient Index Data $(p<0.01)$, EQ-5D $(0=0.02)$, EQ VAS $(p<0.01)$ and Patient Global Assessment $(p<0.01)$. However, none of these were statistically significant at the mid-term (final) assessment $(p>0.05)$.

\section{Other measures}

No data was reported on measures including anxiety and depression, fear avoidance, kinesphophia, self-efficacy, absenteeism or presenteeism measured.

\section{Discussion}

Face-to-face MDT rehabilitation interventions may reduce pain, increase function and improve symptom control for people with MJP, and spa-based treatments may result in short-term reductions in symptoms but have limited longerterm benefits. However, the data were of insufficient quality to provide conclusive evidence of effectiveness because of underpowered cohorts, and limitations in reporting of the diagnostic criteria of MJP, intervention procedures or outcome measures used. It is, therefore, not possible to form recommendations on what interventions or packages of care should be used for people with MJP.

Lack of detailed reporting of interventions is a common limitation in rehabilitation trials [32]. Consequently, interventions which have demonstrated effectiveness cannot be readily adopted into clinical practice, and researchers are unable to replicate, develop and synthase research findings. Only one study in this review involving an MDT programme [31] satisfied the criteria for completeness of intervention reporting according to the TIDieR checklist [15]. Detailed reporting is particularly important for the trials of MJP where the interventions proposed are frequently packages of care which are multi-componented, and offer a range of potential interventions, dosage and settings for delivery. Accordingly, researchers and journal reviewers/editors should be mindful of this when reporting and preparing papers for publication to ensure future trials better describe these interventions.

Conducting this review was complicated by the lack of standardised diagnostic criteria and terminology for people with MJP. This may be partly attributed to the nature of MJP as it represents a complex mix of osteoarthritis, back pain and soft tissue disorders [5]. The most commonly adopted term in the current literature is 'generalised osteoarthritis' $[30,31]$, but other terms include 'widespread musculoskeletal joint pain' [3, 6, 10], 'multi-joint site pain' [9] and 'multi-site joint pain' [5, 33]. Furthermore, a range of different radiological and clinical diagnostic criteria have been used to define MJP [30]. However, there is still no consensus on how to define or classify MJP [34, 35]. Adoption of an agreed term to define this population and use of standardised diagnostic criteria for MJP for future trials will be imperative to improve reporting and implementation.

Outcome assessment of people with MJP can be particularly challenging because of disparate clinical symptoms including pain, fatigue, atrophy and psychosocial traits, and also the varied number and location of joints affected by pain [5] and functional restrictions [1]. This review highlights the current lack of standardisation in outcome measures used in MJP trials. Given the variability in population characteristics and presentation, routine use of outcome measures which examine the global impact of MJP should be considered, such as the HAQ Disability Index (HAQ-DI) validated by Cuperus et al. [1]. Moreover, given that MJP can affect people across the adult life-span, routine inclusion of outcome tools which assess work-related productivity (presenteeism as well as absenteeism) such as the Work Productivity and Activity Impairment Questionnaire [36] would also be valuable for future trials. 
Table 5 Research design considerations for the design of trials on MJP

Standardised definition and criteria for determining the presence of MJP where participants present with two or more joint 'regions' concurrently painful

Trials should record and document the location and frequency of joint sites involved for each trial participant

Interventions aim to treat all painful joint sites participants present with rather than individual joints

Dose, frequency and any modification or tailoring of treatments should be considered to allow flexibility in intervention prescription given the

heterogeneity in the MJP population's symptoms. Decision-making for adaptation of interventions should be presented

All co-interventions should be reported for both experimental or control intervention trial-arms and decision-making for when co-interventions are prescribed should be considered

Outcome measures should be selected to evaluate global health status (symptoms and function) rather than site-specific outcomes

Outcome reporting should be catagorised accordingly to the location and number of joint pain sites participants present with

This study has two limitations of note. First, due to the limited evidence, it was not possible to answer the original research question on the effectiveness of interventions for MJP. Second, due to the variability in diagnostic criteria used and poor study reporting, the review team found the identification of eligible papers a challenge. Strategies including regular discussions on eligibility, member-checking and consensus adjudicators were used to overcome this. Nonetheless, until there is an agreed diagnostic terminology used to classify this population, future systematic reviewers may face similar difficulties. Given the limitations in reporting the diagnostic criteria for MJP and the interventions which have been investigated, we have developed research design considerations (Table 5) to aid the future development of trials which are urgently required to better treat this clinical population.

In conclusion, there is insufficient literature to make clinical recommendations on the treatment of people with MJP. The current evidence-base is limited by study design, diagnostic classification, selection of standardized outcome measures and reporting of study interventions. Given the significant health burden which MJP has on both the individual and wider society, developing and testing interventions to improve symptom management of this condition is a research priority. The methodological considerations highlighted on design and reporting should be considered when developing such trials.

Acknowledgements SRK and PGC are funded in part by the Arthritis Research UK Experimental Osteoarthritis Treatment Centre (Grant number 20083) and the National Institute for Health Research (NIHR) Leeds Biomedical Research Centre. BD is funded by a NIHR Clinical Doctoral Research Fellowship (CDRF-2013-04-044). This article presents independent research funded by the NIHR. The views expressed are those of the authors and not necessarily those of the UK National Health Service (NHS), the NIHR or the UK Department of Health.

Author contributions Conceptualisation: PC, SK. Search strategy and information gathering: TS, RR, BD, CC. Data extraction: RR, BD, CC. Critical Appraisal: BD, CC. Data analysis: TS, BD, CC. Writingoriginal draft preparation: TS, CC, BD, RR, SK, PC. Writing-review and editing: TS, CC, BD, RR, SK, PC. Agreement with manuscript results and conclusions: TS, CC, BD, RR, SK, PC. Guarantor: PC.

\section{Compliance with ethical standards}

Conflict of interest None to declare for any author in relation to this paper.

Ethical approvals Research ethics approval was not necessary to conduct this study design.

Availability of data and materials The dataset supporting the conclusions of this article is included within the article as additional files.

Open Access This article is distributed under the terms of the Creative Commons Attribution 4.0 International License (http://creativecommons.org/licenses/by/4.0/), which permits unrestricted use, distribution, and reproduction in any medium, provided you give appropriate credit to the original author(s) and the source, provide a link to the Creative Commons license, and indicate if changes were made.

\section{References}

1. Cuperus N, Mahler EA, Vliet Vlieland TP, Hoogeboom TJ, van den Ende CH (2015) Measurement properties of the Health Assessment Questionnaire Disability Index for generalized osteoarthritis. Rheumatology 54:821-826

2. Maniadakis N, Gray A (2000) The economic burden of back pain in the UK. Pain 84:95-103

3. Carnes D, Parsons S, Ashby D, Breen A, Foster NE, Pincus T, Vogel S, Underwood M (2007) Chronic musculoskeletal pain rarely presents in a single body site: results from a UK population study. Rheumatology 46:1168-1170

4. Allison TR, Symmons DP, Brammah T, Haynes P, Rogers A, Roxby M, Urwin M (2002) Musculoskeletal pain is more generalised among people from ethnic minorities than among white people in Greater Manchester. Ann Rheum Dis 61:151-156

5. Raja R, Dube B, Hensor EMA, Hogg SF, Conaghan PG (2016) The clinical characteristics of older people with chronic multisite joint pains and their utilization of therapeutic interventions: data from a prospective cohort study. BMC Musculoskelet Disord 17:194

6. Lillefjell M, Krokstad S, Espnes GA (2006) Factors predicting work ability following multidisciplinary rehabilitation for chronic musculoskeletal pain. J Occup Rehabil 16:543-555 
7. Hopman-Rock M, Odding E, Hofman A, Kraaimaat FW, Bijlsma JW (1997) Differences in health status of older adults with pain in the hip or knee only and with additional mobility restricting conditions. J Rheumatol 24:2416-2423

8. Lacey RJ, Belcher J, Rathod T, Wilkie R, Thomas E, McBeth J (2014) Pain at multiple body sites and health-related quality of life in older adults: results from the North Staffordshire Osteoarthritis Project. Rheumatology 53:2071-2079

9. Finney A, Healey E, Jordon JL, Ryan S, Dziedzic KS (2016) Multidisciplinary approaches to managing osteoarthritis in multiple joint sites: a systematic review. BMC Musculoskelet Disord 17:266

10. Moradi B, Zahlten-Hinguranage A, Barié A, Caldeira F, Schnatzer P, Schiltenwolf M, Neubauer E (2010) The impact of pain spread on the outcome of multidisciplinary therapy in patients with chronic musculoskeletal pain-a prospective clinical study in 389 patients. Eur J Pain 14:799-805

11. Raja R, Kingsbury SR, Wise E, Conaghan PG (2014) Primary care approaches to musculoskeletal multiple-site joint pain pharmacological therapy: a survey of general practitioners. Prim Health Care Res Dev 15:476-481

12. NICE (2014) National Institute for Health and Care Excellence. Osteoarthritis: care and management. CG177. Published. Accessed: 22.09.2016. http://www.nice.org.uk/guidance/cg177/ chapter/About-this-guideline

13. Rosemann T, Joos S, Laux G, Gensichen J, Szecsenyi J (2007) Case management of arthritis patients in primary care: a clusterrandomized controlled trial. Arthritis Rheum 57:1390-1397

14. van Baar ME, Dekker J, Oostendorp RA, Bijl D, Voorn TB, Bijlsma JW (2001) Effectiveness of exercise in patients with osteoarthritis of hip or knee: nine months' follow up. Ann Rheum Dis 60:1123-1130

15. Hopman-Rock M, Westhoff MH (2000) The effects of a health educational and exercise program for older adults with osteoarthritis for the hip or knee. J Rheumatol 27:1947-1954

16. Bellamy N, Buchanan WW, Goldsmith CH, Campbell J, Stitt LW (1988) Validation study of WOMAC: a health status instrument for measuring clinically important patient relevant outcomes to antirheumatic drug therapy in patients with osteoarthritis of the hip or knee. J Rheumatol 15:1833-1840

17. Roos EM, Roos HP, Lohmander LS, Ekdahl C, Beynnon BD (1998) Knee Injury and Osteoarthritis Outcome Score (KOOS)development of a self-administered outcome measure. J Orthop Sports Phys Ther 28:88-96

18. Fairbank JC, Couper J, Davies JB (1980) The Oswestry Low Back Pain Questionnaire. Physiotherapy 66:271-273

19. Ware J Jr, Kosinski M, Keller SD (1996) A 12-Item Short-Form Health Survey: construction of scales and preliminary tests of reliability and validity. Med Care 34:220-233

20. Ware JE Jr, Sherbourne CD (1992) The MOS 36-item short-form health survey (SF-36). I. Conceptual framework and item selection. Med Care 30:473-483

21. Herdman M, Gudex C, Lloyd A, Janssen M, Kind P, Parkin D, Bonsel G, Badia X (2011) Development and preliminary testing of the new five-level version of EQ-5D (EQ-5D-5L). Qual Life Res 20:1727-1736
22. Zigmond AS, Snaith RP (1983) The hospital anxiety and depression scale. Acta Psychiatr Scand 67:361-370

23. Waddell G, Newton M, Henderson I, Somerville D, Main CJ (1993) A Fear-Avoidance Beliefs Questionnaire (FABQ) and the role of fear-avoidance beliefs in chronic low back pain and disability. Pain 52:157-168

24. Miller RP, Kori S, Todd D (1991) The Tampa Scale: a measure of kinesiophobia. Clin J Pain 7:51-52

25. Schwarzer R, Jerusalem M (1995) Generalized self-efficacy scale. In: Weinman J, Wright S, Johnston M (eds) Measures in health psychology: a user's portfolio. Causal and control beliefs. Windsor, pp 35-37

26. Downs SH, Black N (1998) The feasibility of creating a checklist for the assessment of the methodological quality both of randomised and non-randomised studies of health care interventions. J Epidemiol Community Health 52:377-384

27. Hoffmann TC, Glasziou PP, Boutron I, Milne R, Perera R, Moher D, Altman DG, Barbour V, Macdonald H, Johnston M, Lamb SE, Dixon-Woods M, McCulloch P, Wyatt JC, Chan AW, Michie S (2014) Better reporting of interventions: template for intervention description and replication (TIDieR) checklist and guide. BMJ 348:g1687

28. Higgins JPT, Green S (2011) Cochrane handbook for systematic reviews of interventions version 5.1.0 [updated March 2011]. The Cochrane Collaboration. http://www.handbook.cochrane. org. Accessed 19 Oct 2017

29. Slavin RE (1995) Best evidence synthesis: an intelligent alternative to meta- analysis. J Clin Epidemol 48:9-18

30. Erol FB, Forestier RJ, Guneri FD, Karagulle MZ, Erdogan N (2014) Spa therapy for generalized osteoarthritis: an open, observational, preliminary study. Therapie 70:273-281

31. Cuperus N, Vliet Vlieland TP, Mahler EA, Kersten CC, Hoogeboom TJ, van den Ende CH (2015) The clinical burden of generalized osteoarthritis represented by self-reported health-related quality of life and activity limitations: a cross-sectional study. Rheumatol Int 35:871-877

32. Yamato TP, Maher CG, Saragiotto BT, Hoffmann TC, Moseley AM (2016) How completely are physiotherapy interventions described in reports of randomised trials? Physiotherapy 102:121-126

33. de Cássia Pereira Fernandes R, da Silva Pataro SM, de Carvalho RB, Burdorf A (2016) The concurrence of musculoskeletal pain and associated work-related factors: a cross sectional study. BMC Public Health 16:628

34. Dougados M, Nakache JP, Gueguen A (1996) Criteria for generalized and focal osteoarthritis. Rev Rhum Engl Ed 63:569-575

35. Forestier R, Francon A, Briole V, Genty MC, Chevalier X, Richette P (2011) Diagnostic criteria for generalized osteoarthritis: a preliminary study in a population with knee osteoarthritis. Jt Bone Spine 78:424-426

36. Dibonaventura MD, Gupta S, McDonald M, Sadosky A, Pettitt D, Silverman S (2012) Impact of self-rated osteoarthritis severity in an employed population: cross-sectional analysis of data from the national health and wellness survey. Health Qual Life Outcomes 10:30 